\title{
Aprendizaje de la escritura en lengua no materna: Casos de niños tarahumaras
}

\section{Learning of Writing in a Non-Mother Tongue: the Case of Tarahumara Children}

\author{
Efrén Viramontes Anaya ${ }^{l}$ \\ Escuela Normal Rural "Ricardo Flores Magón" \\ Saucillo, Chih. México \\ Correo electrónico: efren8000@hotmail.com \\ Lylia Ana Morales Sifuentes ${ }^{2}$ \\ Escuela Normal Rural "Ricardo Flores Magón" \\ Saucillo, Chih. México \\ jesusm0124@hotmail.com \\ Luis Manuel Burrola Márquez \\ Escuela Normal Rural "Ricardo Flores Magón" \\ Saucillo, Chih. México \\ luisburrola1@yahoo.com.mx
}

Recibido 01 de diciembre de 2010 • Aceptado 09 de marzo de 2011

Resumen. Esta investigación analiza el desarrollo de la escritura en español de niños indígenas tarahumaras bilingües (del Estado de Chihuahua, México), desde varias perspectivas: la psicogenética, relacionada con el avance en el proceso de construcción del sistema de escritura de los niños investigados; la social, mediante al análisis del contexto familiar, cultural y el entorno del asentamiento de convivencia de los mismos; y la pedagógica, aunque más breve, a través del ambiente áulico dentro de dos escuelas muy diferentes: una regular y otra indígena. El planteamiento central de la investigación giró en torno a la percepción de los múltiples factores que se relacionan con el aprendizaje de la escritura, en un intento de escudriñar analíticamente los elementos posibles de afectación en el proceso referido. La metodología cualitativa utilizada posibilitó, mediante el estudio de casos, la observación, la entrevista, la videograbación y el análisis de los cuadernos de los niños, percibir situaciones y rescatar evidencias que, mediante el estudio transversal de eventos, personas y contextos, dieron como resultado interpretaciones sobre los factores sociales, culturales, cognitivos y pedagógicos que se percibieron asociados al aprendizaje de la escritura de una lengua que no es la materna, en niños indígenas migrantes establecidos en un medio distinto al de sus ancestros. Se atiende principalmente a la hipótesis de que, en las circunstancias de los casos estudiados, es más conveniente que aprendan a escribir primero en su segunda lengua y, posteriormente, en la materna, si así lo requieren.

Palabras claves. Escritura, indígena, aprendizaje, Tarahumaras.

\footnotetext{
Doctorado en Ciencias de la Educación por el Instituto Pedagógico de Estudios de Posgrado en Celaya, Gto. México. Labora como docente en la Universidad Pedagógica Nacional de Delicias, Chih. México, en las asignaturas de metodología de la investigación. También es docente investigador de tiempo completo en la Escuela Normal Rural "Ricardo Flores Magón” de Saucillo, Chih, México.

2 Docente investigador de tiempo completo en la Escuela Normal Rural "Ricardo Flores Magón” de Saucillo, Chih, México.

3 Docente investigador de tiempo completo en la Escuela Normal Rural "Ricardo Flores Magón” de Saucillo, Chih, México.
} 
Abstract. This study analyzes the development of writing in Spanish among bilingual Tarahumara children (Chihuahua, Northern Mexico), from different perspectives: 1) A psychogenetic perspective, focusing on how the writing process is constructed by Tarahumara children; 2) A social perspective, focusing on the children's family context, as well as the cultural and economic characteristics of their community, and; 3) A pedagogical perspective, focusing on the learning environments of two different kinds of schools these children attend (a "regular" school and a bilingual school for indigenous children). This study tries to identify how different factors have an impact on the development of writing. Data was collected for this multiple-case study through on-site observation, interviews and videotaping class sessions at both schools. Children's notebooks and writing products were also collected. The data was analyzed transversally considering events, people and contexts related to the writing process. The results were interpretations on the social, cultural, cognitive and pedagogical factors that were perceived, associated to the learning process of how to write in a non-mother tongue, in the case of migrant indigenous children, in an environment different from that of their ancestors. The study was based on the hypothesis that, in the circumstances of the studied cases, it is better for these children to learn first how to write in their second language and, then, in their mother tongue, if necessary.

Keywords. Writing, indigenous, learning, Tarahumaras.

\section{Introducción}

El análisis de las dificultades que tienen los niños tarahumaras y sus docentes en el aprendizaje y enseñanza del sistema de escritura constituye un tema de estudio que cobra importancia en el sentido de aportar una explicación, a partir de estudios de caso, de lo que sucede en dicho proceso cuando los que aprenden no pertenecen al contexto sociocultural ni lingüístico del lugar en que lo hacen.

También tiene una importancia particular el hecho de observar las dificultades que los docentes tienen al atender un grupo "regular" y a otro multigrado de niños tarahumaras, en dos escuelas totalmente diferentes en una misma localidad semirural.

El planteamiento principal gira en torno a las dificultades de los alumnos tarahumaras, de ambas escuelas, en el aprendizaje de la escritura en español. Los ámbitos a considerar son: el trabajo de los niños, de los docentes y el contexto familiar y social del asentamiento indígena.

La realización de esta investigación aporta niveles de comprensión de las causas por las cuales existen esas dificultades en los docentes y en los estudiantes tarahumaras al momento de construir, desde cada escuela, el sistema de escritura del español.

El análisis de la construcción del sistema de escritura por parte de los niños tarahumaras se realizó a partir de la aportación de un esquema explicativo, a la luz de los avances teóricos que existen sobre el tema de estudio, desde dos perspectivas: una psicogenética y otra sociocultural.

Los resultados puedan ser extrapolados a otros contextos, como un modelo interpretativo del proceso seguido en la investigación y las aportaciones al horizonte teórico de este tema de estudio, en el sentido de la comprensión más generalizada del proceso de aprendizaje de la lengua escrita en un idioma que no es el materno y cuando este último es indígena.

Derivado de las precisiones anteriores, el problema de investigación se planteó de manera concreta como sigue: ¿Qué factores se asocian al aprendizaje del sistema de escritura del español de los niños tarahumaras migrantes? 


\section{Objetivo general:}

Analizar la situación de los niños migrantes indígenas en el proceso de aprendizaje del sistema de escritura en una lengua que no es la materna.

\section{Objetivos específicos:}

- Analizar y detectar factores implicados en el proceso de la construcción de la lengua escrita de los niños tarahumaras (primer ciclo de la educación primaria)

- Conocer dificultades de los niños y los docentes en el proceso de construcción de la escritura

- Conocer las aportaciones o limitaciones del contexto familiar, cultural y social para el aprendizaje de la escritura.

- Generar elementos de insumo para propuestas aplicables a niños indígenas migrantes.

\section{Metodología de la investigación}

La metodología utilizada para esta investigación es de tipo cualitativa. Una primera parte se realizó a través del estudio de "método de casos", con la participación de cuatro niños tarahumaras; y la otra mediante el análisis de los elementos contextuales y familiares del asentamiento indígena y de las dos escuelas primarias (regular e indígena). Los apoyos metodológico fueron: Gagneten (1987), Martínez (2005) y Stake (2007).

El análisis de casos aportó valiosa información sobre el proceso de construcción del sistema de escritura de cada niño, en relación con los diferentes factores que se asocian al mismo. Posterior a esto se realizó un análisis transversal de los casos, junto con los elementos del contexto social, familiar y cultural de los indígenas, haciendo referencia a la construcción del sistema de escritura del español, utilizando categorías de análisis rescatadas por las aportaciones teóricas consultadas sobre la temática.

Las categorías de análisis utilizadas fueron:

- Proceso de construcción del sistema de escritura de los niños tarahumaras

- La condición de aprender a escribir en una lengua o idioma que no es el materno

- La influencia del contexto familiar y social en el aprendizaje de la escritura del español como segundo idioma

- El ambiente de interacción social del grupo escolar y la generación de oportunidades específicas de aprendizaje

- Expectativas académicas de los tarahumaras

- Exigencias académicas de la escuela indígena y la regular

- El aprovechamiento de la interacción social en el grupo por parte del docente en el aprendizaje de la escritura

- Función del albergue en el desarrollo académico de los niños tarahumaras

- Problemas de enseñanza de los docentes que atienden a los niños tarahumaras. 
En general, las aportaciones derivadas del análisis mediante las categorías transversales permitieron interpretar y generalizar los hallazgos de la investigación, extrapolando los resultados hacia los ámbitos en los que existan niños migrantes obligados a aprender a escribir en otro idioma.

Los 4 casos, en esta investigación, están representados por 3 niños tarahumaras del grupo de primer grado de la escuela primaria indígena: Ana, María y Martín ${ }^{4}$. El cuarto caso es Petra, de la escuela primaria regular ${ }^{5}$, quien después del análisis de los resultados, se constituyó en un parámetro de comparación con los otros niños, por las características específicas que guarda el caso y que se describen en el texto.

El análisis de la información del proceso de construcción de la escritura se realizó a través de categorías establecidas por el enfoque teórico de este tema de estudio que proponen Ferreiro y Teberosky (2007), Ferreiro y Gómez (1991), Gómez (2000) y Nemirovsky (2004). Los elementos contextuales y relacionados con el aprendizaje y enseñanza de la escritura se basaron en las aportaciones de Vigil (s. f.), Schmelkes (2002, 2005 y 2010) y Echeverría (2001).

Las técnicas de acopio de información son la observación y la entrevista. La primera se realizó en 16 sesiones de primer grado, en la asignatura de Español, relacionadas con la enseñanza y aprendizaje de la lengua escrita. Se focalizó en las acciones que realizan los niños tarahumaras y en las actividades didácticas o de intervención pedagógica que realiza el docente en la enseñanza de la escritura. Los datos que se obtuvieron aquí son evidencias de avance de construcción del sistema de escritura en los niños, las actividades didácticas del docente, las dificultades de los alumnos y maestros, así como elementos del contexto familiar social de los niños implicados.

También fue motivo de observación el contexto socioeconómico, familiar y cultural en el que se desenvuelven los alumnos tarahumaras. Los datos obtenidos son características, descripciones y evidencias de la influencia del contexto en la formación de los alumnos.

La entrevista se realizó a once personas: cuatro a niños tarahumaras para encontrar sentido e información sobre el proceso de construcción de la lectura; cuatro más a padres de familia, para obtener datos del contexto, las actividades familiares y de apoyo a la educación de los niños, así como de otros problemas o asuntos relativos con el desarrollo del sistema de escritura de sus hijos; dos entrevistas a los maestros de los grupos de primer grado de la escuela primaria, con el propósito de indagar sobre las dificultades de enseñanza, de aprendizaje y de las acciones concretas que realizan para sortear dichas dificultades; la undécima entrevista se efectuó al encargado del albergue de Rosales, Chih., en el que se encuentran asistidos los niños tarahumaras del asentamiento indígena.

También se realizaron análisis de los trabajos y cuadernos de los niños, para observar el avance y dificultades que tienen en la construcción del sistema de la escritura del español. Eventualmente, junto con el análisis de los cuadernos y trabajos, se efectuó una entrevista clínica a los niños para comprender los niveles de avance que tienen en la construcción de la escritura.

En todos los casos de las entrevistas se obtuvieron datos similares a los de las observaciones, mismos que sirvieron para triangular y complementar información obtenida entre las diversas fuentes.

La información generada en el proceso de la investigación de los casos estudiados, los eventos de clases de las dos escuelas, las entrevistas y las observaciones del contexto, se trataron transversalmente con categorías de análisis que permitieron la triangulación de los datos dentro de un patrón de sentido determinado por dichas categorías.

$4 \quad$ Los nombres de los casos fueron cambiados para proteger su identidad, por la misma razón se eliminó el nombre de las escuelas primarias y de la localidad en la que se encuentran.

5 Es una escuela primaria común y normal a la que asisten la mayoría de los estudiantes mexicanos. 


\section{Resultados}

\section{A. Avance de la escritura de los casos y de la generalidad de los niños tarahumaras}

El proceso de construcción del sistema de escritura, no solo del Estado de Chihuahua, sino también de los Estados de la república mexicana, se desarrolla de manera más sistemática durante el primer grado de la escuela primaria. En la generalidad de los niños, llegan a construirlo en el nivel "alfabético" entre los 6 y 7 años de edad. Sin embargo, los niños migrantes tarahumaras, además de ingresar a la educación formal de la escuela primaria entre los 7 y 8 años, se tardan, en promedio, dos años más para apropiarse del sistema en ese mismo nivel.

Por lo tanto, es común que se observe en los asentamientos indígenas de los diferentes municipios del estado de Chihuahua que los niños tarahumaras, en su mayoría, acceden al nivel alfabético alrededor de los 9 años de edad, dos años más tarde que los niños "chabochis" (forma en que denominan a las personas mestizas) de las mismas comunidades en las que se encuentran asentados.

En la información recabada durante la investigación se pudo constatar la diferencia existente entre los niños de la escuela primaria indígena y los de la escuela primaria regular, con respecto al aprendizaje de la escritura. Los niños de la escuela regular avanzan más rápido en la construcción de este proceso. Los factores que se involucran dependen más del tipo de escuela y del contexto en que viven (economía, cultura étnica, familia y política), que de los procesos individuales de los niños.

Los niveles de construcción del sistema de escritura del español en los niños analizados en los cuatro estudios de caso fueron (ver tabla 1):

Tabla 1

Niveles de escritura de los 4 casos

\begin{tabular}{llll}
\hline Alumno & Escuela & Edad & Nivel \\
Petra & Regular & 6 años 10 meses & Silábico alfabético \\
Ana & Indígena & 7 años 6 meses & Silábico alfabético \\
María & Indígena & 8 años 3 meses & Silábico \\
Martín & Indígena & 8 años 11 meses & Presilábico \\
\hline
\end{tabular}

En coincidencia con las expectativas, las diferencias en los resultados de los alumnos de la escuela primaria indígena y la primaria regular son significativas, considerando las edades y los avances que tienen los niños de cada grupo, como se indica en la tabla 2:

Tabla 2

Comparativo de niveles de escritura entre las dos escuelas investigadas

\begin{tabular}{|l|c|c|c|c|c|c|c|c|c|}
\hline \multirow{2}{*}{ Escuela } & \multirow{2}{*}{ No. de alumnos } & \multicolumn{2}{|c|}{ Nivel alfabético } & \multicolumn{2}{|c|}{ Nivel silábico alfabético } & \multicolumn{2}{|c|}{ Nivel silábico } & \multicolumn{2}{|c|}{ Nivel presilábico } \\
\cline { 3 - 10 } & & Abs. & $\%$ & Abs. & $\%$ & Abs. & $\%$ & Abs. & $\%$ \\
\hline Regular & 24 & 21 & 87,5 & 1 & 4,16 & 2 & 8,33 & 0 & 0 \\
\hline Indígena & 12 & 0 & 0 & 1 & 8,33 & 5 & 41,66 & 6 & 50 \\
\hline
\end{tabular}


Lo que se observa en las tablas anteriores es la evidencia de que los niños tarahumaras tienen niveles de aprendizaje del sistema de escritura del español mucho más bajos que los niños "chabochis" de la misma población. Los factores asociados con la tardanza y las problemáticas para aprender la escritura son de tipo cultural, social y contextual, además de aquéllos que se relacionan con el ambiente de la escuela y los procesos metodológicos que se emplean en la misma.

\section{B. La condición de aprender a escribir en una lengua o idioma que no es el materno}

El aprendizaje de la escritura en un idioma que no es el materno, como el caso que analizamos en esta investigación, aprender a escribir en español teniendo como lengua materna al tarahumara, es una condición especial dentro del sistema educativo mexicano. En lo general, existen sugerencias en los sistemas de educación bilingües de que se aprenda a escribir primero a partir de la lengua materna, para utilizarla como base y referencia para construir el sistema de escritura de la segunda, sin considerar, como señala Vigil (s. f.) y Schmelkes (2002), que la lenguas indígenas son ágrafas, es decir, originalmente no tienen escritura, son generadas y reproducidas por tradición oral. No tienen grafías especiales que se hayan diseñado por el mismo grupo indígena para realizar su impresión y representación.

El tarahumara, como lengua, se escribe con las grafías que se utilizan en el español, esto se hizo inicialmente por mestizos o por tarahumaras que aprendieron a escribir primero en español. El proceso se realiza a través del principio de correspondencia grafofonética, haciendo la transpolación de la escritura hacia el tarahumara, utilizando, la mayoría de las veces, las reglas gramaticales de este último. En este proceso se toman decisiones sobre convencionalidades ortográficas arbitrarias en la escritura de las palabras, ya que es más frecuente el uso de las letras $\mathrm{K}$ y W que en el idioma español.

Esto nos conduce a pensar que la enseñanza de la escritura en los niños tarahumaras debe hacerse primero en español por parte del sistema escolar indígena bilingüe, tal y como se está haciendo en la escuela primaria indígena investigada.

\section{La influencia del contexto familiar y social en el aprendizaje de la escritura del español como segundo idioma}

El contexto familiar de Petra es adecuado o favorecedor para el aprendizaje de la escritura del español por varios motivos. Sus padres saben leer y escribir. La mamá de Petra realizó estudios de educación secundaria y los concluyó. Situación poco común dentro de la historia de escolaridad de los habitantes del asentamiento de indígenas tarahumaras de Rosales, principalmente porque tanto hombres como mujeres se casan o se "juntan" con sus parejas a muy temprana edad, justo en la que se encontrarían cursando la educación secundaria, en caso de haber tomado la decisión de hacerlo.

Los padres de Martín y María no terminaron la educación primaria, solo la mamá de Ana lo hizo, pero no convive con ella. Por lo tanto, el ambiente en el que viven es muy poco favorecedor para su vida escolar.

La mamá de Petra lee cuentos con su hija, le presenta frecuentemente actos de lectura y tienen convivencia académica. Sabe exactamente de los avances de la lectura y la escritura, le ayuda, aunque no se percata que lo hace erróneamente, desde el punto de vista metodológico, toda vez que 
interviene haciéndole los trabajos, diciéndole el nombre de las letras en lugar de trabajar de una manera más constructiva. Es muy evidente que, en el contexto familiar de Petra, existen esfuerzos serios y sistemáticos para que aprenda a escribir lo mejor posible. No así en las familias de Martín, Ana y María.

El papá de Petra apoya completamente la decisión que tomó la mamá de enviarla a la escuela regular, en lugar de mandarla a la escuela indígena, por considerar que aprenderá más en aquella, aunque esta última se encuentre enfrente de su casa. Los padres de Martín, María y Ana ni siquiera pusieron a consideración la opción de la escuela primaria regular para que estudiaran sus hijos.

Existe una preocupación constante de los padres para que a Petra no le falte nada en la escuela. La mamá asiste periódicamente a la institución, o cuando se le requiere, para tratar asuntos relacionados con su hija. Le provee de los materiales y útiles escolares que necesita. Aporta las cuotas escolares.

La mamá de Petra no trabaja fuera de su hogar, como las madres de Martín, Ana y María. Esta situación le permite atender mejor las necesidades que tiene Petra en la escuela y en sus procesos de aprendizaje. Situación que difiere de los otros casos.

La familia se percibe estable en el sentido de que los padres siempre están en su casa; es decir, no suelen retirarse para realizar trabajos en ranchos lejanos como lo hace la familia de Martín y otros tarahumaras, mismos que se ausentan de sus casas hasta por varios meses.

Aunque Martín, Ana, Petra y María han nacido ya en un contexto en el que el español es una lengua de uso frecuente, por la necesidad de utilizarla en el contexto social en el que viven; sin embargo, la emisión verbal castellana no es tan eficiente en sus padres y familiares como para usarla con la precisión y fluidez que lo hace cualquier otro niño mestizo.

Pinto y Sorribes (1996, p. 234) citan que diversas investigaciones demuestran que los niños, con un vínculo de afecto positivo y estable en el seno familiar, resultan con mayores habilidades sociales. Este es el caso de Petra, de quien se percibe un buen ambiente familiar, así como una preocupación por parte de sus padres para que su desarrollo académico sea más alto que el del promedio de los niños indígenas del asentamiento. En lo casos restantes, existe alejamiento, como los padres de Martín por su trabajo y de la mamá de Ana por vivir separada de la familia. Estos distanciamientos familiares son derivados de la situación económica que viven.

En este estudio de casos se realizaron algunas actividades para conocer el contexto familiar y social que rodea a los alumnos focalizados: se asistió a las casas de cada uno de ellos, se entrevistó a padres y familias que se encuentran alrededor de la escuela.

Esta cultura se caracteriza por vivir en familias extensas, es decir, la familia nuclear (padre, madre e hijos) cohabita con abuelos, hermanos, sobrinos, tíos y toda la parentela cercana que se encuentra en la comunidad, comparten la misma casa y los mismos servicios sanitarios. En las diferentes visitas al asentamiento se pudo constatar, a través de las entrevistas y observaciones realizadas, que los niños (casos) no permanecen en el núcleo familiar durante los cinco días hábiles de la semana, tiempo durante el cual se encuentran en el albergue, en las condiciones que se describen más adelante.

De los cuatro casos investigados, solamente la madre de Petra tiene escolaridad de secundaria. En los casos de los padres de Martín, María y Ana, sólo la madre de esta última asistió a la escuela primaria, los demás no saben leer ni escribir. En todos los casos los padres son jornaleros (tienen diferentes empleos temporales) y las madres, excepto la de Petra, se dedican al trabajo en el campo agrícola, también de manera temporal, en la cosecha del chile, cebolla y nuez que se realiza en los alrededores del asentamiento, es decir, ellas no salen de la comunidad en la que viven, mientras que los padres regularmente trabajan en temporadas fuera de ella. 
En el contexto del asentamiento, los habitantes se comunican regularmente en su dialecto, aunque también usan el español; las personas son, en lo general, retraídas y poco comunicativas con los "chabochis", razón por la cual al principio de la investigación se dificultó un poco la comunicación; no obstante, con las diferentes visitas que se realizaron al contexto, se logró que la interacción fuera más fluida, de tal manera que posibilitó la realización de las entrevistas y pláticas informales con las familias.

Por la misma forma en la que conviven los miembros de las familias tarahumaras, es difícil que conserven evidencias de los avances que los niños tienen en la escuela. En algunos casos ni siquiera saben que existen las boletas de calificaciones ni su significado, porque la contextualización e interacción con el medio escolar es muy escasa o nula. El último contacto que se tuvo fue con la familia de Martín, ahí pudimos constatar que efectivamente la madre del niño desconocía el avance escolar. Cabe destacar que esta plática fue al término del ciclo escolar y esta madre no sabía si el niño leía o no, si había aprobado o reprobado el ciclo escolar: la conversación en la familia giró en torno a la falta de comunicación que se genera con la misma escuela y los padres de familia. Aquí cabe subrayar que el maestro que atiende la escuela primaria indígena también es de origen tarahumara, por lo tanto, la cultura de retraimiento y poca comunicación es característica también de él.

Los niños tarahumaras solamente conviven en el asentamiento con niños de su propia etnia, dentro de la escuela indígena y en el albergue; la única niña que está dentro de un grupo distinto es Petra, quien asiste a una escuela regular. Sin embargo, esa distinción no ha sido del todo favorecedora para su desarrollo académico, como se describirá más adelante.

\section{El ambiente de interacción social del grupo escolar genera oportunidades específicas de aprendizaje}

En las observaciones que se realizaron en los salones de clase, se pudo percibir que Petra se relaciona con sus compañeros con muchas restricciones. Por lo general es retraída, no participa libre y voluntariamente si la maestra no la obliga a hacerlo.

Petra se ve en el grupo como una alumna diferente. Es una niña tarahumara, la única en el grupo. La carga histórica de discriminación y relegación de la raza se refleja de manera muy clara en las relaciones que tiene entre pares dentro del grupo y en los juegos en el recreo.

El grupo del primer grado de la escuela primaria regular ofrece a Petra dos situaciones contradictorias entre sí, con respecto a la valoración del clima grupal en el que estudia. La primera situación es que, al ser diferente, se ve limitada en su desarrollo interpersonal y social. Está descontextualizada con respecto al tipo de compañeros que tiene. Ninguno de sus compañeros del albergue está en su grupo. Se percibe que exhibe vergüenza y miedo para participar en clases.

La interacción social que tiene no es tan favorable como la que se observa en el resto de los compañeros de su grupo. No existe relación entre pares para la realización o comentarios que habitualmente hacen sobre los trabajos o actividades escolares. Se ve como una isla dentro del grupo. Esta situación se interpreta como negativa para la generación de oportunidades de aprendizaje derivadas del ambiente de las interacciones sociales dentro del grupo escolar al que pertenece.

La segunda situación, que es contraria a esta primera, es que la exigencia de trabajo académico en la escuela primaria regular es más alta en relación con la exigencia generada en la escuela primaria indígena. Esta situación ha permitido que Petra haya avanzado más en el ámbito de la construcción de la escritura del español que María, Martín y Ana, quienes son alumnos de la escuela primaria indígena. Sin embargo, ha perdido la oportunidad de convivir con sus iguales de raza, con sus amistades de barrio y del albergue. 
En otras palabras, se ha preferido el hecho de tener un mejor avance académico que un mejor desarrollo personal y social de Petra en la interacción y adaptación como persona, con una identidad tarahumara bien definida, pero soslayada en aras de que tenga mejores oportunidades de trascender académicamente, por sobre los promedios de niveles máximos de estudio establecidos en las personas del asentamiento indígena.

Por su parte, los casos de Martín, Ana y María, aunque viven una interacción más abierta entre compañeros, no es lo suficientemente fuerte para resarcir las deficiencias generadas por la intervención didáctica del docente ni de las carencias del contexto familiar, social y cultural en el que se desenvuelven.

Finalmente, cada centro educativo, tanto el regular como el indígena, ofrecen a los niños tarahumaras ventajas que no han podido capitalizar para avanzar en el rezago que evidentemente tienen con respecto a los demás niños de la población.

\section{E. Expectativas académicas de los tarahumaras}

Las expectativas académicas de los tarahumaras migrantes son más bajas que las de los habitantes de la localidad en la que habitan. Esta situación se refleja, en general, no solo en los diferentes asentamientos indígenas, según resultados de la Evaluación Nacional del Logro Académico de los Centros Escolares (ENLACE) del 2010 (SEP, 2010), en todos los municipios del estado de Chihuahua, sino también en el ámbito de los resultados nacionales de México.

En lo que se recogió de información en esta investigación se pudo constatar que los padres de los niños tarahumaras los envían a la escuela primaria entre los siete u ocho años, mientras que los chabochis lo hacen a entre los seis y siete. Esto refleja desconocimiento de las edades en las que los niños deben o pueden ingresar a la primaria por parte de los adultos tarahumaras o la combinación de esta situación con la percepción de que los niños de 6 años les parecen "físicamente" muy pequeños para que asistan a la escuela.

Los logros académicos que tradicionalmente han tenido las tarahumaras y los indígenas, en general, han sido bajos (Instituto Nacional de Estadística y Geografía [INEGI], 2005), en relación con el resto de la población mexicana. No han percibido la necesidad de la realización de estudios formales de acuerdo con su cultura o se rinden ante la difícil posibilidad de atender los requerimientos económicos e intelectuales que les exige la escuela formal.

Los pocos niños tarahumaras que han ingresado a realizar estudios de la educación secundaria se han visto truncados por: la necesidad de trabajar para contribuir económicamente con la familia; otros han tenido dificultades para solventar los gastos que implican los materiales, uniformes y demás requerimientos; otros más se casan a la edad en la que están cursando este nivel educativo. Estas causas se presentan también de manera combinada, es decir, que es frecuente que dos o más de estas situaciones se presentan simultáneamente como característica de los niños que se aventuran a estudiar la secundaria.

Por otra parte, Echeverría (2001) sostiene que existe una falta de cobertura muy pronunciada en educación secundaria para los indígenas de Chihuahua (aunque también en las demás niveles educativos y entidades federativas del país); señala que "ha faltado voluntad política para que las comunidades indígenas accedan al nivel de secundarias... En total se lleva el servicio educativo a una población de 20,552 niños y niñas indígenas en edad escolar en las modalidades de educación inicial, preescolar, primaria y en forma limitada a secundaria" (p. 43-44), esta es una de las principales limitantes que los tarahumaras han tenido para acceder a la educación secundaria. 
Existen espacios en las escuelas secundarias regulares, pero no los hay en especial para los indígenas, como sí es el caso del preescolar y la primaria. Para poder estudiar la secundaria tienen que ingresar a la escuela regular, con una alta posibilidad de fracaso. Otra opción que es más frecuentemente utilizada por los indígenas es la educación abierta para adultos.

El último elemento de análisis de esta situación, pero que también tiene que ver con el aprendizaje de los niños, es el estado físico con el que se enfrentan al proceso de aprendizaje. Con respecto a este tema, Echeverría (2001) señala que: "la prevalencia de déficit de talla en los niños refleja la deficiencia nutricional de las poblaciones indígenas, mientras que el 59\% de los niños en primarias indígenas presenta déficit de talla, sólo el 17,4\% de los niños en las primarias regulares y el 2,8\% de las primarias privadas se encuentran en esta situación" (pp. 34-35). Esta condición, además de provocar retraso en el aprendizaje, hace que los adultos tarahumaras perciban que a los 6 años los niños son todavía muy pequeños para ingresar a la primaria.

Todo este panorama, en el que las tradiciones culturales, las condiciones económicas, las exigencias institucionales, las condiciones familiares y las oportunidades reales para que los indígenas realicen estudios superiores a la educación primaria, hace que las expectativas académicas de los niños migrantes tarahumaras sean mucho más bajas que las de la población en la cual se encuentra su asentamiento. Ante esta situación, lo establecido en el artículo $11^{\circ}$ de la Ley de Derechos Lingüísticos de los Pueblos Indígenas (Poder ejecutivo de los Estados Unidos Mexicanos, 2003) y lo establecido como garantía en el Artículo $2^{\circ}$ Constitucional (de México), no se cumplen a cabalidad y quedan solo como una buena intención.

\section{F. Exigencias académicas de la escuela indígena y la escuela regular}

Las exigencias académicas en la escuela primaria regular suelen ser más altas que en la escuela indígena. Esto se da porque en la escuela regular tiene un docente por cada grupo-grado. Por ejemplo, en la primaria regular, en donde estudia Petra hay seis grupos, uno por cada grado de primero a sexto. Cada grupo tiene un maestro que lo atiende de manera exclusiva; es decir, no atiende a otro grupo más que ese. El número de alumnos que tiene en promedio es de 24.

La escuela primaria regular tiene maestro de Educación Física, un equipo de la Unidad de Servicios de Apoyo a la Escuela Regular (USAER) para atender los problemas de aprendizaje y a los niños con necesidades educativas especiales. La maestra de grupo recibe asesoramiento técnico pedagógico en el aula (por lo menos la de primer grado).

Cuando se aborda un contenido curricular en la escuela regular, se hace con alumnos que se encuentran en un estatus con características más cercanas a la homogeneidad que a la heterogeneidad, situación que no se vive en el grupo multigrado de la escuela indígena.

Estas condiciones generan un ambiente de trabajo con un buen grado de exigencia, es decir, no se entretiene tanto con la diversidad en los alumnos. Los temas o contenidos curriculares son únicos, salvo algunas posibles excepciones de niños con necesidades educativas especiales. Los niños se concentran más en lo que hacen. El docente ofrece un trato más centrado en el trabajo que se realiza de manera casi uniforme en el grupo. No se dispersa tanto la actividad y atención del docente.

Por su parte, la escuela primaria indígena tiene generalmente esa misma cantidad de alumnos en el grupo, sólo que este contiene alumnos de los seis grados. Es un grupo multigrado.

El docente del grupo indígena no tiene apoyos de USAER ni apoyos técnicos pedagógicos en el aula o, por lo menos, cercanos o accesibles geográficamente a la escuela. Tampoco se imparten, aquí, cursos de Educación Física, ni Educación Artística. El ambiente de trabajo es más disperso y 
complejo, para atenderlo se requiere de una alta capacidad pedagógica y de un trabajo de planeación más fino, que le exige al docente un alto conocimiento y dominio de los programas de estudio.

Los niños tienen más dificultad con respecto a sus marcos referenciales para abordar los contenidos curriculares. Los útiles escolares que usan son más restringidos que en la escuela regular, debido a que el nivel socioeconómico de los padres es más bajo.

Existen problemas más pronunciados de impuntualidad, inasistencia, de cumplimiento de tareas, de acercamiento de los padres de familia a la escuela y de apoyo extraescolar de los mismos.

En general, se puede concluir que la exigencia académica de la escuela primaria regular es mucho más alta que la que se percibe en la escuela primaria indígena, aun cuando en esta última se tengan apoyos de computadora, impresora, libros, materiales diversos de la Secretaría de Educación Pública (SEP) y aire acondicionado en el salón de clases.

\section{G. El aprovechamiento de la interacción social en el grupo por parte del maestro, facilita el aprendizaje de la escritura}

La maestra de Petra dice que tiene problemas para que participe en clase. Difícilmente provoca que interaccione con sus compañeros de grupo. En ocasiones tiene que dirigirse a ella específicamente para lograr una participación en clase, mientras los demás alumnos del grupo lo hacen de manera espontánea.

Esta situación, más que facilitar el aprendizaje por interacción social, a Petra le perjudica, toda vez que en este sentido tiene restricciones o limitaciones para interactuar con sus compañeros, aun en los eventos en los que ellos podrían ayudar en su aprendizaje, o en los que se requiera de trabajo en equipo, ponerse de acuerdo, discutir temas o contenidos curriculares.

La maestra de grupo interviene deliberadamente para que Petra interactúe con sus compañeros, la invita a participar y la cuestiona directamente, pero sus esfuerzos han sido poco fructíferos. Después de un año de trabajo grupal no ha podido establecer un clima en el que Petra pueda desenvolverse por lo menos a nivel promedio de los demás alumnos.

Los niños de la escuela indígena, aunque tienen una excelente interacción entre iguales dentro del salón de clases, es una condición que no es aprovechada por el docente, tal vez por la carga extra que le significa el trabajar con un grupo multigrado o por falta de habilidad didáctica.

Perret-Clemont (1980), citado por Cazden (1991), menciona, como característica favorecedora para la formación integral de los niños, que "la interacción entre iguales intensifica el desarrollo del razonamiento lógico" (p. 140). Con respecto a esto, en el caso de Petra se percibe desfavorecida, toda vez que el ambiente de la clase en la escuela donde asiste le restringe la interacción con sus compañeros, por la condición de ser de otra raza. La conducta de interacción de Petra con sus compañeros pareciera que no es entre iguales. No existe la confianza suficiente para desplegar un tipo de relación que favorezca el aprendizaje que habitualmente se logra entre los integrantes de un grupo escolar. Esta situación no se presenta en los casos de Martín, Ana y María, porque la relación con sus compañeros se muestra totalmente abierta.

Según Mondragón y Trigueros (2002): "Los profesores han de esforzarse por prestar atención a estos procesos interactivos tratando de asegurar que se producen en la dirección de crear y fomentar relaciones de aceptación, ayuda mutua, cooperación y respeto por las diferencias" (p. 28); en este sentido, la maestra de Petra refiere que trata constantemente de que participe con los demás compañeros y que lo haga individualmente también ante el grupo. Sin embargo, dice que es difícil para Petra, porque se cohíbe y se retrae constantemente ante esta situación. 
Johnson (1981), citado por César Coll (1984), describe tres formas básicas de organización social de las actividades, denominadas: cooperativa, competitiva e individualista. Con relación con esto, en el caso de Petra, la maestra se preocupa porque ella participe en actividades individuales; sin embargo, el trabajo con la forma básica de organización llamada cooperativa, generaría en ella oportunidades de interacción con sus iguales, mismas que le permitirán un desarrollo académico y de integración social más completo. La forma de trabajo cooperativo es una de las debilidades encontradas en los indígenas de la escuela regular, pero no en los casos de Martín, Ana y María, ni en lo general de la escuela indígena.

Lo pertinente, desde la perspectiva del Plan de Estudios de Educación Primaria 2009, es que estos asuntos de la diversidad y la interculturalidad se trabajen a través de todas las modalidades de asignaturas, como un contenido transdisciplinario, que aplica igualmente a todo tipo de escuela de educación básica (Secretaría de Educación Pública [SEP], 2009).

\section{H. Funciones del albergue en el desarrollo académico de los tarahumaras}

El sistema de albergues, como al que se hace referencia en este estudio, representa una alternativa que ayuda a evitar el fracaso escolar de los niños del asentamiento indígena que se investiga. Este enunciado encierra la importancia de dicha institución con respecto a su función de asistencia y apoyo a la educación de grupos vulnerados.

En lo particular, el albergue al que asisten los niños tarahumaras del asentamiento estudiado cumple principalmente la función de brindarles servicios asistenciales que en sus casas tal vez no tendrían, o por lo menos no en las mismas condiciones como las que se les ofrecen en esta institución. Estos servicios consisten en darles hospedaje, alimentación e higiene diaria. Esto hace posible que no exista pretexto para faltar a la escuela o para que su aprendizaje se vea afectado por falta de alimentación.

Otro elemento que aporta el alberque es el apoyo académico que se les brinda a los niños para hacer sus tareas escolares, con el acompañamiento de un "tutor" o maestro que apoya, cuando es necesario, para que cumplan con sus trabajos académicos sin pretexto alguno.

El tutor es una persona habilitada para el trabajo académico en educación primaria. Son dos maestros que en un principio fueron contratados con perfil de bachillerato, pero que se les exigió, por parte de los Servicios Educativos del Estado de Chihuahua (SEECH), que realizaran estudios de licenciatura en educación.

Se considera que el apoyo de los tutores no es especializado. Es decir, como principio no son maestros de educación primaria bilingüe. Solo uno de ellos está en proceso de realización de sus estudios en este campo y está cursando la Licenciatura en Educación Intercultural. El otro tutor estudia licenciatura en Educación Secundaria que, aunque es del campo de la educación, no se forma para atender con suficiente solvencia teórica el proceso de adquisición del sistema de escritura en el nivel de educación primaria.

Así, la deficiencia más notable de los tutores es que, con respecto al tratamiento didáctico del sistema de escritura, no se percibe el conocimiento del marco de referencia metodológica más avanzado en el ámbito pedagógico ni del enfoque pedagógico del Plan de estudios 2009; toda vez que cuando se trata de las tareas de escritura se observa que el apoyo se basa más en el copiado de palabras que en el seguimiento y desarrollo del proceso psicogenético de los niños en la construcción del sistema de escritura, como lo proponen Ferreiro y Teberovsky (2007), Ferreiro y Gómez (1991), Gómez (2000) y Nemirovsky (2004). 
Una prueba de ello se infiere en lo que comenta el director del albergue con respecto a Petra, niña del albergue y alumna de la escuela primaria regular, cuando señala que: "Petra iba muy bien.... cuando salió del kínder... copiaba todo muy bien, pero se atrasó, no sé por qué", se resalta la palabra "copiaba" porque eso indica la forma en que apoyan las actividades de enseñanza y aprendizaje de la escritura del español, esto refleja parte de la metodología utilizada por los tutores. Con María y Ana, se aplica la misma forma de apoyo en la escritura. En el caso de Martín, refieren que se ausenta por periodos largos, porque su papá se lo lleva a trabajar, con él tienen menos oportunidad de apoyarlo.

Los apoyos que el albergue oferta a los niños indígenas del asentamiento y de otros lugares circunvecinos son: clases de Educación Física, Educación Artística, manualidades y otras actividades.

En términos generales, aun con los inconvenientes que se señalaron con respecto a los perfiles profesionales de los tutores, el servicio que brinda el albergue de Rosales es indispensable para que los niños no deserten de la escuela primaria, que asistan con regularidad y que sean bien alimentados.

\section{Problemas de enseñanza de los docentes que atienden a los niños tarahumaras}

Se detectaron problemas de enseñanza (según lo establecido en la bibliografía especializada ya referida en este documento), percibidos a través de las observaciones efectuadas en las aulas y de los trabajos de los niños de la escuela primaria regular e indígena.

En la escuela regular, se detectó mayor "alejamiento" metodológico en la primera maestra, de las dos que atendieron el grupo. Esto se pudo inferir mediante los trabajos que les encargó a los niños durante las primeras semanas de clases de primer grado. Un primer argumento de la inconsistencia metodológica se percibió en que los trabajos evidenciaban sólo copia de palabras del pizarrón o de hojas de ejercicios impresas y previamente preparadas.

El segundo argumento se tiene a partir de los ejercicios de "caligrafía" que les encargaba que realizaran. El tercer argumento se observó en las actividades que, en general, se infirieron de los cuadernos de los niños en las cuales no se reflejaba la metodología propuesta por el programa escolar. En todas las clases se le dio más importancia a la actividad mecánica que a las acciones que permitieran la construcción del sistema de escritura del español.

La segunda maestra trabajó de manera más apegada al programa oficial, por lo tanto sus intervenciones pedagógicas se consideran más adecuadas que las de su antecesora. Esto contribuyó para que más de 20 alumnos del grupo concluyeran su ciclo escolar 2009-2010 con mayor nivel en el proceso de construcción del sistema de escritura y más competentes en la comunicación escrita.

En lo que respecta a la intervención del docente de la escuela indígena, se repite la historia de la primera docente que tuvieron los niños de la escuela regular. Las evidencias que se tienen de las observaciones y de los trabajos de los niños en sus cuadernos, indican que las intervenciones didácticas para favorecer el proceso de construcción del sistema de escritura del español en los niños tarahumaras no eran las más adecuadas.

En dichas intervenciones se infiere que utiliza la copia de palabras, los ejercicios de caligrafía y actividades de relleno y entretenimiento. Esto último, tal vez, debido a la complejidad de atender a un grupo multigrado en el que conviven cotidianamente alumnos de los seis grados de la educación primaria. Se observó que la atención del maestro era más atraída por los alumnos de los grados superiores. 
Con todo lo anterior, se concluye que las intervenciones de los docentes que se involucraron en los procesos de aprendizaje del sistema de escritura de los niños tarahumaras migrantes, requieren de actualización para mejorar la intervención didáctica en la escritura del español y del tarahumara. También deben considerar y aprovechar las condiciones de los grupos. Aun así, se perciben dos fortalezas:

La primera se presenta en la escuela primaria regular: es el hecho de tener un solo grado por maestro y alumnos con marcos de referencia más adecuados para el desarrollo de sus niveles de construcción de la escritura. Además de la disposición de los contextos familiares que apoyan a los niños en el aprendizaje de la expresión escrita en particular.

La segunda tiene que ver con la escuela primaria indígena: las condiciones que se observaron de armonía de los tarahumaras al estudiar con sus "iguales" y no en una escuela regular con niños "chabochis", donde generalmente son retraídos, con muy poca interacción y poco participativos.

En el caso de la escuela indígena, el docente requiere de usar más lo recursos que tienen en el aula y mejorar el sistema de organización en el grupo multigrado. Se requiere, además, que exista sensibilidad, respeto y mejor atención por parte de las autoridades educativas para generar mejores condiciones de atención educativa para este sector tan vulnerado.

\section{Discusión y conclusiones}

Los principales resultados apuntan hacia la develación de la condición problemática que tienen los niños de aprender a escribir en un idioma que no es el materno. Se resaltan una serie de factores que se encuentran asociados a las dificultades que los niños tarahumaras tienen para aprender a escribir en español. Estos factores tienen que ver con los procesos individuales de los mismos, sus familias, el contexto del asentamiento, exigencias de las escuelas primarias, las metodologías de los docentes, los climas de trabajo en los grupos escolares, las expectativas académicas de los tarahumaras y los libros de texto que se utilizan.

Con respecto a los libros de texto, existen de dos tipos en la escuela indígena, los que se utilizan en el sistema educativo nacional de la escuela regular y los que están escritos en lengua tarahumara. El problema de los primeros es que están elaborados para cultura mestiza, ajenos a la realidad contextual de los niños indígenas con respecto a su cultura de origen.

Los libros editados especialmente para la etnia Tarahumara tienen la desventaja de que muchas de las palabras no las entienden los niños por las variedades lingüísticas que existen en las regiones de la sierra Tarahumara, de donde provienen sus progenitores. Esto coincide con las conclusiones que al respecto tiene Schmelkes (2002), cuando señala que: "Entre las comunidades hay pequeñas variaciones linguiísticas, tanto de pronunciación como de vocabulario. La identidad comunitaria, vinculada a la lengua, conduce a rechazar como propia una lengua que [...] difiere ligeramente de la forma utilizada en la comunidad propia" (p. 12). Por lo tanto, es importante establecer que existe concordancia con esta misma especialista, con respecto a que los libros de texto que se utilizan en las comunidades indígenas tienen limitaciones de identidad lingüística en el uso estandarizado con todos los grupos tarahumaras.

El clima de trabajo en las aulas donde estudian los tarahumaras, tanto en escuelas regulares como indígenas, es diferente en cada una de ellas, pero ambas contienen características que, si no son conscientemente reconsideradas por los docentes, resultan inadecuadas para el aprendizaje de los niños.

Donde estudia Petra, la escuela primaria regular, el clima de trabajo entre pares "chabochis" es bueno, se tienen evidencias de observaciones y gráficas de la forma en que se desarrolla el trabajo áulico. En este sentido, la interacción que se da es exitosa, pero no para Petra que se muestra escindida 
y cohibida con respecto al trato cotidiano con sus compañeros del grupo. Esta es una característica típica de los indígenas cuando conviven en contextos o espacios de los no indígenas. Por lo que se percibe una deficiencia en la planificación del trabajo docente que pueda resarcir esta situación.

El clima de trabajo en la escuela regular es más exigente que en la escuela indígena, pero la interacción entre pares se ve más favorecida en la escuela indígena; por lo tanto, para los niños tarahumaras las interacciones entre iguales son más formativas, en su aspecto integral como personas, y poco aprovechado por el docente para el aprendizaje de la escritura del español.

El ambiente familiar es un factor determinante para el ingreso, permanencia y aprendizaje en la escuela primaria. Existen ambientes más alfabetizadores que otros, aun entre los tarahumaras. Como conclusión, aunque parezca obvio, el ambiente de las familias de los mestizos es más alfabetizador que el de los tarahumaras.

En las calles por las que transitan habitualmente los niños desde el albergue hasta la escuela primaria y los alrededores de su casa, existen muy pocos portadores de textos visibles que les permitan considerar la escritura como parte del medio en el que viven. En este sentido, el contexto general no es un ambiente que favorezca la apropiación del sistema de escritura en los niños.

El contexto del asentamiento indígena es poco alfabetizador, esto coincide con la idea de Ferreiro y Teberosky (2007), quienes plantean que existen medios en los que no hay una relación directa con textos escritos, es decir, que no se exhiben letreros o anuncios gráficos como en los lugares más urbanizados: "en sus juguetes, en los carteles publicitarios o en los carteles indicadores, en su ropa, en la TV, etc." (p. 29); sin embargo, en estos casos, el medio semiurbano marginado, más cercano o parecido al rural, no le aporta tanto como en un medio completamente urbanizado. Aunado a esto, los maestros de las escuelas regular e indígena tienen diferencias en las exigencias académicas que caracterizan las actividades de enseñanza. La escuela indígena es muy poco exigente en cuanto a la realización de estrategias didácticas y modalidades de trabajo para generar aprendizajes significativos. Esta situación hace que los niños tarahumaras transiten más lentamente por sus procesos de construcción del sistema de escritura del español.

Se percibe, en los trabajos y actividades de los niños, un enfoque metodológico más favorecedor en la escuela primaria regular (en la que comúnmente asisten alumnos y maestros mestizos). Se observa mayor problema de enseñanza en la escuela indígena, esto sucede en la mayoría de ellas, según lo que señala Schmelkes (2010) en la caracterización de las escuelas y docentes de educación indígena de México. Comenta que en estas, los docentes indígenas generalmente son contratados sin un perfil profesional educativo. Después de obtener el trabajo de docentes se habilitan como tales; pero con la actitud de que al obtener la plaza lo toman como “(...) el punto de llegada, no el punto de partida para una carrera profesional (...)" (p. 217); concluye con la idea de que "(...) tampoco están dotados de los elementos necesarios para ofrecer una educación de calidad (...)" (p. 217).

Las dificultades en el aprendizaje de los niños tarahumaras, migrantes establecidos, se perciben como determinadas por los factores familiares, contextuales, culturales y académicos de sus escuelas y maestros. Además, no se aprovechan los medios y recursos de la escuela indígena como: enciclomedia ${ }^{6}$, biblioteca, computadora, impresora, proyector y materiales escolares.

El albergue al que asisten los niños del asentamiento tarahumara ofrece servicios asistenciales que previenen o evitan la inasistencia o ausentismo escolar y las posibilidades de fracaso escolar por falta de apoyo asistencial como alimentación y hospedaje. En el ámbito académico cumple la función de apoyar la realización de las tareas escolares. Se percibe deficiencia en el perfil y desempeño profesional de las tutoras.

Equipo de cómputo y software electrónico de apoyo a la educación primaria de México 
El nivel máximo de estudios de la población tarahumara es el de primaria completa e incompleta, eventualmente algunos estudian y concluyen la educación secundaria. Su cultura, costumbre y tradiciones hacen que los jóvenes tarahumaras se casen en edad en que deberían estar cursando la educación secundaria (14 a 15 años).

Realmente son muy pocos los tarahumaras que tienen una visión de futuro, en la que la educación secundaria, media y profesional forme parte de sus vidas. Esta forma de pensar no necesariamente es mala, pero no ayuda a la idea de preocuparse por avanzar académicamente como una forma de culturalización alterna, necesaria para supervivencia en su nuevo contexto social, paralelo a su cultura de origen.

El idioma tarahumara que hablan los niños del asentamiento no es tan fluido y rico como el de sus padres y abuelos, pero sí les es posible comunicarse con ellos en el ámbito doméstico y cotidiano. Esta condición los limita también, porque en su contexto se utilizan los dos idiomas, pero ninguno desarrollado a profundidad, es decir, no hablan el español como lo hacen sus compañeros del grupo, ni el tarahumara como lo hacen sus padres y abuelos.

En la mayoría de los tarahumaras se observa un problema de sintaxis en el lenguaje oral. Esta es definida como: "Parte de la gramática que enseña a coordinar y unir las palabras para formar las oraciones (...) La que establece el enlace de las palabras de la manera más lógica y sencilla." (Acanomas, 2010). Tienen dificultades para enlazar las palabras en la conformación de enunciados. Generalmente no coinciden con la convencionalidad de los usos que hacen de género y número. Esta condición afecta, de alguna manera, la estructuración de la escritura de los niños con una correspondencia global sonoro-gráfica con sentido.

El avance del aprendizaje del sistema de escritura en español es más lento en los niños indígenas que en los mestizos, mientras que estos últimos lo aprenden en promedio entre los 6 y 7 años, los tarahumaras lo hacen entre los 7 y 9, con mucho más dificultad. Sobre todo si el apoyo de los docentes no es el adecuado, desde el punto de vista metodológico.

El problema del aprendizaje de la escritura en una lengua que no es la materna, reside principalmente en que se tienen pocos referentes del contexto familiar y social que sirvan de base y soporte para la construcción del sistema de escritura. Es decir, al tener como lengua materna la indígena y el hecho de utilizarla en sus hogares y contexto inmediato, además de la poca participación que tienen con los diferentes ámbitos sociales no indígenas (chabochis), provoca la dificultad, a los niños tarahumaras (y de cualquier otra etnia), para establecer asociaciones gráficas concretas (portadores de textos en español) y frecuentes con el idioma español y los principios gramaticales que lo caracterizan.

Con respecto a la pertinencia de enseñarles a escribir primero en español (lengua no materna), en lugar de hacerlo en su lengua indígena (materna) tiene sus detractores, entre ellos se encuentra la idea que presenta Schmelkes (2002), cuando señala:

Sabemos que es conveniente enseñar a leer y a escribir en la lengua materna. Por evidente que esto resulta - no es posible aprender a leer y a escribir en una lengua que uno no entiende- los maestros en sus prácticas no siempre muestran compartir este principio. La segunda lengua puede comenzarse a enseñar oralmente, y una vez que se muestran avances en los aspectos receptivos y creativos de la lengua oral (comprensión y expresión) puede enseñarse en forma escrita (p. 15). 
La tesis contraria a la de Schmelkes, que se establece como resultado de esta investigación, es que en el caso de los niños migrantes indígenas, inclusive los que están en sus lugares de origen, les es muy difícil escribir en una lengua que tradicionalmente no se ha escrito por parte de sus ancestros, por ser ágrafa. No posee estructura gramatical que la soporte. Esto hace sumamente difícil la enseñanza y el aprendizaje de la escritura. La lengua indígena ágrafa debe permanecer como tal, y cultivarse como tradicionalmente se ha hecho: a través de la oralidad, esto cobra sentido solo en parte de la propuesta de Schmelkes (2002), cuando sugiere que la escuela debe ser un espacio público de uso de la lengua indígena en su modalidad original (oral). La escuela, entonces, debe convertirse en un espacio de convivencia y aprendizaje intercultural.

Enseñarles primero a escribir en español podría parecer como una agresión cultural hacia los niños indígenas, sin embargo, se tienen que considerar varias situaciones con las cuales se argumenta lo contrario. Primero, es necesario establecer que los niños indígenas migrantes, como a los que se hace referencia en esta investigación, no se encuentran ya en sus contextos originales de su etnia tarahumara. Esto los lleva a que inevitablemente tengan que convivir con gente "chabochi" que está establecida en la población o comunidad a la que ahora pertenecen. Esta decisión, que fue tomada por sus padres o sus abuelos, es una realidad que tienen que enfrentar o sobrellevar, tratando de obtener de ella lo mejor para su desarrollo como ciudadanos, considerando también su identidad como tarahumaras.

Si ya están establecidos en el contexto que no es el origen de su etnia, y además si se considera que estos niños ya no regresarán a la sierra tarahumara, implica que se quedarán a vivir en un contexto al cual tienen que adaptarse (en el mejor de los sentidos) sin tener que perder para ello su identidad. En esta tarea, la escuela y la familia juegan un papel importante. Por ello se hace necesario tratar de resolver los problemas del aprendizaje de la escritura en el idioma que no es el materno, pero que es el que les permitirá desenvolverse mejor en el nuevo ambiente social en que vivirán en lo sucesivo. Esta se percibe como una acción indispensable e impostergable, toda vez que el sistema educativo en el que están realizando sus estudios escolarizados está estructurado para que las etnias en general, se desarrollen en su cultura como sociedad e individuos y les provee los elementos necesarios para que también puedan adaptarse a la interacción social con el resto de los ciudadanos mexicanos, con los que comparten el mismo territorio, instituciones, leyes, espacios sociales, de salud y culturales (entre otros).

El principio que se propone para que la educación de los indígenas migrantes sea más relevante y pertinente, en los términos establecidos en las políticas educativas actuales de México, es la promoción efectiva de la educación intercultural (Schmelkes (2002 y 2005), toda vez que los niños se enfrentan con una cultura propia a un proceso de aprendizaje que pertenece a otra cultura (mestiza), misma que no debe ser desdeñada tampoco porque es a la que se están integrando como ciudadanos de una comunidad más amplia que la de su asentamiento o barrio. En este sentido, tendrán mejores oportunidades de participar en los eventos ciudadanos, con derechos y obligaciones, así como en la exigencia de promover las compensaciones de atención generadas por la relegación histórica de la que han sido objeto, respetando el fomento de sus costumbres y tradiciones.

Quedan pendientes, para continuar con la investigación, los análisis de las diferentes propuestas de intervención que se sugieren para la atención de la enseñanza y aprendizaje de la escritura, tanto del español (o lengua no materna), como de la lengua indígena (lengua materna), en grupos de indígenas migrantes que van a la ciudad y regresan constantemente a sus lugares de origen; además de aquellos que permanecen en sus localidades sin emigrar a otros espacios sociales en los que el idioma y la cultura pueden o no ser compartidas con la de los mestizos a través de la convivencia obligada del espacio geográfico común. 


\section{Referencias bibliográficas}

Acanomas. (2010). Diccionario español. [Sintaxis] Recuperado de http://www a.acanomas.com/ Diccionario-Espanol/128186/SINTAXIS.htm

Cazden, B. (1991). El discurso en el aula. El lenguje de la enseñanza y del aprendizaje. Barcelona: Ediciones Paidós.

Coll, C. (1984). Estructura grupal, interacción entre alumnos y aprendizaje escolar. Infancia $y$ aprendizaje. $\mathrm{N}^{\circ}$ 27-28, 119-138. Recuperado de http://dialnet.unirioja.es/servlet/ articulo?codigo $=668449$

Echeverría G., H. (2001). La educación indígena en Chihuahua; hacia la construcción de la interculturalidad. En H. Echeverría (Coord.), Antología asignatura regional I ( pp. 42-46). Chihuahua, México: Secretaría de Educación y Cultura SEP.

Ferreiro, E. y Gómez, M. [Comps.]. (1991). Nuevas perspectivas sobre los procesos de lectura y escritura ( $8^{\mathrm{a}}$ ed.). Buenos Aires: Siglo Veintiuno editores.

Ferreiro, E. y Teberosky, A. (2007). Los sistemas de escritura en el desarrollo del niño (23ª ed.). México: Siglo veintiuno editores.

Gagneten, M. (1987). Hacia una metodología de sistematización de la práctica. Buenos Aires: Editorial Hvmanitas

Gómez, M. (2000). El proceso de adquisición del sistema de escritura. En M. Gómez y A. Martínez (Coords.), La adquisición de la lectura y la escritura en la escuela primaria (pp. 135-140). México: Secretaría de Educación Pública (SEP).

Instituto Nacional de Estadística y Geografía [INEGI]. (2005). Conteo nacional de población y vivienda 2. México: Autor.

Martínez, M. (2005). Actualización de la epistemología y metodología en educación. En III Jornada de Innovación Educativa. Universidad Centroccidental Lisandro Alvarado, Barquisimeto, Edo. Lara. Recuperado el 29 de abril del 2009 en http://www.ucla.edu.ve/viacadem/redine/ jornadas/III\%20Jornadas\%20REDINE/PonenciasdelasIIIJornadas.htm

Mondragón, J. y Trigueros, I. (2002). Intervención con menores. Acción socioeducativa. España: Narcea. Nemirovsky, M. (2004). Antes de empezar: ¿qué hipótesis tienen los niños acerca del sistema de escritura? [Anexo 2]. En E. Moreno (Coord.). Modulo III. Aproximación de los niños al lenguaje escrito (pp. 41-47). México: SEP. Recuperado de http://www.oei.es/linea3/ ModuloIII.pdf

Pinto, V. y Sorribes, S. (1996). El aula como contexto social: las relaciones entre iguales. En R. A. Clemente y C. Hernandez (Coords.), Contextos de desarrollo psicológico y educación (pp. 233-237). Granada: Aljibe. 
Poder ejecutivo de los Estados Unidos Mexicanos. (2003). Ley general de derechos lingüísticos de los pueblos indígenas. México. Recuperado de http://www.diputados.gob.mx/LeyesBiblio/ pdf/257.pdf

Secretaría de Educación Pública [SEP]. (2009). Evaluación nacional de logro académico de los centros escolares (ENLACE). México. Recuperado de http://www.enlace.sep.gob.mx/ba/ db2010/estadisticas2.html

Schmelkes, S. (octubre, 2002). La enseñanza de la lectura y la escritura en contextos multiculturales. Conferencia presentada en el VII Congreso Latinoamericano para el Desarrollo de la Lectura y la Escritura, Puebla, México. Recuperado de www.amdh.com.mx/ocpi/documentos/ docs/6/15.doc

Schmelkes, S. (enero, 2005). La interculturalidad en la educación básica. Conferencia presentada en el Encuentro Internacional de Educación Preescolar: Currículum y competencias, organizado por Editorial Santillana, México. Recuperado de http://www.amdh.com.mx/ocpi/ documentos/docs/6/16.pdf

Schmelkes, S. (2010). Indígenas rurales, migrantes, urbanos: una educación equivocada, otra educación posible. Pensamiento Iberoamericano, N 7, 203-222. Recuperado de http://www. pensamientoiberoamericano.org/xnumeros/PensamientoIbero7.pdf

Stake, R. (2007). Investigación con estudio de casos. Madrid, España: Morata.

Vigil, N. (s. f.). Pueblos indígenas y escritura. Portal aulaintercultural. Recuperado de http://www. aulaintercultural.org/IMG/pdf/indigenas escritura.pdf 